\title{
FOLIATIONS IN ALGEBRAIC SURFACES HAVING A RATIONAL FIRST INTEGRAL
}

\author{
Alexis García Zamora
}

\begin{abstract}
Given a foliation $\mathcal{F}$ in an algebraic surface having a rational first integral a genus formula for the general solution is obtained. In the case $S=\mathbb{P}^{2}$ some new counter-examples to the classic formulation of the Poincaré problem are presented. If $S$ is a rational surface and $\mathcal{F}$ has singularities of type $(1,1)$ or $(1,-1)$ we prove that the general solution is a non-singular curve.
\end{abstract}

\section{Introduction}

A foliation $\mathcal{F}$ in a complex algebraic surface $S$ is a non-identically zero morphism of vector bundles $\alpha: L \rightarrow T S$, where $L$ is a line bundle and $T S$ is the tangent bundle of $S$. In the case $S$ is the projective plane, there are alternative definitions in global terms and, in particular, there is a notion of degree of a foliation (see Definition 1). Explicitly, the degree of $\mathcal{F}$ is the degree of the polynomials $Y_{i}$, where the direction field

$$
Y=\sum_{i=0}^{2} Y_{i}\left(x_{0}: x_{1}: x_{2}\right) \frac{\partial}{\partial x_{i}}
$$

defines the foliation.

In [10] Poincaré studied the following problem: if $\mathcal{F}$ is a foliation with a rational first integral (that is, all the solutions of $\mathcal{F}$ are algebraic curves) is it possible to bound the degree of the generic solution in terms of the degree of $\mathcal{F}$ ? An answer to this problem gives a "practical" method to determine when a foliation has a rational first integral. If such a 
bound exist then the existence of a first integral is "simply" a problem of algebraic calculus.

The answer to this problem is well know to be no. In section 2 some counter-examples are presented. Our first task is then to reformulate the Poincaré problem. A reasonable formulation is: try to bound the degree of the general solution using information depending only of $\mathcal{F}$, for example the eigenvalues of the singularities of $\mathcal{F}$ (we assume that all the singularities of $\mathcal{F}$ are of multiplicity one).

In section 1 we present preliminary results and definitions. All this material can be found in $[\mathbf{6}]$, $[\mathbf{9}]$ or $[\mathbf{1 0}]$.

In section 2 we study the version of the problem presented above. The section starts with the following:

Proposition 2.1. Let $\mathcal{F}$ be a foliation on $S$ with a first integral and singular points of multiplicity one. Denote the pair of relatively prime integers associated to the eigenvalues of the nodes of $\mathcal{F}$ by $\left(u_{i}, v_{i}\right)$, then we have:

$$
\begin{gathered}
\sum_{i=1}^{n} r_{i}\left(u_{i}+v_{i}\right)=-C \cdot\left(K_{S}+L\right), \\
g=-\frac{C . L}{2}-\frac{\sum r_{i}}{2}+1 .
\end{gathered}
$$

Where $C$ is a general solution of $\mathcal{F}$,

$g=$ geometric genus of $C$,

$\alpha: L \rightarrow T S$ defines $\mathcal{F}$,

$K_{S}=$ canonical divisor of $S$,

$r_{i}=$ number of branches of $C$ through a node of $\mathcal{F}$,

$n=$ number of nodes of $\mathcal{F}$.

Then the counter-examples to the old formulation of the Poincaré problem are presented; Example 2.1 is well know, Examples 2.2 and 2.3 seem new. In particular Example 2.2, the pencil $y^{d}=\lambda x^{d-2}(x-1)(y-1)$, proves that the genus of the pencils of curves giving the counter-examples can be arbitrary. Some more specific versions of the Poincaré problem are then presented.

We finish the section by showing that if $m=\operatorname{deg} \mathcal{F}>4$ and for some cases if $m \leq 4$ a bound for the genus of the generic solution is sufficient in order to solve the reformulated Poincaré's Problem. Then we introduce the study of certain linear series on the general solution and the concept of linearly equisingularity and prove the following: 
Proposition 2.3. If the conditions of being linearly equisingular to $C$ imposes independent conditions on curves of degree $d$ then

$$
g(C) \leq \delta
$$

Where $\delta=\operatorname{deg}\left(\mathcal{O}_{\tilde{C}}(\tilde{C})\right)$, and $\tilde{C} \stackrel{\pi}{\rightarrow} C$ is the minimal embedded resolution of the general solution $C$.

In section 3 we study the foliations in $\mathbb{P}^{2}$ having as first integral a generic pencil. The main aim is to prove:

Theorem 3.4. Let $\mathcal{F}$ be a irreducible foliation in a rational surface $S$ with singularities of eigenvalues $(1,1)$ or $(1,-1)$. If $\mathcal{F}$ has a rational first integral $f: S \rightarrow \mathbb{P}^{1}$, then all the fibers of $f$ are reduced curves and the general one is a non-singular curve meeting transversally any other fiber of $f$.

An important corollary is:

Corollary 3.5. Let $\mathcal{F}$ be a foliation in $\mathbb{P}^{2}$ of degree $m$ with a first integral of degree $d$, if all the singular points of $\mathcal{F}$ have eigenvalues $(1,1)$ or $(1,-1)$ then

$$
m=2 d-2 .
$$

This section contains a generalization of some results of Poincaré [10]. Propositions 3.1, 3.2 and 3.3 were proved by Poincaré for the case $S=\mathbb{P}^{2}$. Even when Poincaré knew that under the hypothesis of Corollary 3.5 the degree of $m$ is bounded, an explicit bound is not given in [10].

I want to thank CIMAT support and to X. Gómez-Mont for many useful comments. In particular, the proof of Proposition 3.1 was suggested to me by X. Gómez-Mont and C. Danthony.

\section{Preliminaries}

Definition 1. A foliation $\mathcal{F}$ of degree $m$ in $\mathbb{P}^{2}(\mathbb{C})$ is determined by either of the following objects:

a) An algebraic Pfaff equation given by a projective 1-form

$$
\omega=\sum_{i=0}^{2} \omega_{i}\left(x_{0}: x_{1}: x_{2}\right) d x_{i}=0
$$


with $\omega_{i}$ homogeneous polynomials of degree $m+1$ and

$$
\sum_{i=0}^{2} x_{i} \omega_{i} \equiv 0
$$

b) A direction field

$$
Y=\sum_{i=0}^{2} Y_{i}\left(x_{0}: x_{1}: x_{2}\right) \frac{\partial}{\partial x_{i}}
$$

with $Y_{i}$ homogeneous polynomials of degree $m$, modulo a sum of $G\left(x_{0}: x_{1}: x_{2}\right) R$, where

$$
R=\sum_{i=0}^{2} x_{i} \frac{\partial}{\partial x_{i}}
$$

is the radial vector field, and $G$ is a homogeneous polynomial.

c) A map of vector bundles $\alpha: L_{-(m-1)} \rightarrow T \mathbb{P}^{2}$ where $L_{-(m-1)}=$ $H^{\otimes-(m-1)}$, and $H$ is the hyperplane bundle on $\mathbb{P}^{2}$.

If the greatest common divisor of $\left(\omega_{i}\right)$ is equal to one, $\mathcal{F}$ is called an irreducible foliation.

Definition 2. The singular set $\mathcal{S}$ of $\mathcal{F}$ is determined by:

a) The common zeros of the $\omega_{i}$.

b) The points $\left(x_{0}: x_{1}: x_{2}\right)$ such that

$$
\frac{Y_{0}\left(x_{0}: x_{1}: x_{2}\right)}{x_{0}}=\frac{Y_{1}\left(x_{0}: x_{1}: x_{2}\right)}{x_{1}}=\frac{Y_{2}\left(x_{0}: x_{1}: x_{2}\right)}{x_{2}} .
$$

c) The points $p$ where $\alpha: L_{-(m-1)} \rightarrow T \mathbb{P}^{2}$ is not injective.

The condition g.c. d. $\left(\omega_{i}\right)=1$ is equivalent to the singular set of $\mathcal{F}$ is finite.

The algebraic multiplicity of the singularities is defined alternatively as:

a) In affine coordinates, write a local expression for $\omega$ in terms of homogeneous monomials

$$
\omega=\sum_{k=r}^{\infty} w_{1, k}(x, y) d x+\sum_{k=r}^{\infty} w_{2, k}(x, y) d y
$$


and the first number $r$ such that $w_{1, r}$ or $w_{2, r}$ is not zero is called the algebraic multiplicity of the singularity.

b) Analogous way for the local expression of the direction field.

Moreover, if $\mathcal{F}$ has algebraic multiplicity 1 in a singular point $p$ and the eigenvalues corresponding to the linear part of $\mathcal{F}$ in $p$ are non zero the singularity is called of multiplicity one.

We assume in the following that $\mathcal{S}$ is a discrete set and that the linear part of the associated local vector field is invertible (i.e., all the singularities have multiplicity one).

Definition 3. According with each formulation in Definition 1, a curve $C$ in $\mathbb{P}^{2}$ is called a leaf of $\mathcal{F}$ if:

a) It is a solution of the differential equation associated to $\omega=0$.

b) The tangent line in each point $p$ of $C-\mathcal{S}$ coincides with the line determined by $Y(p)$.

c) For each point $p, \alpha$ send the fibers of $L_{-(m-1)}$ on $p$ into the tangent line of the curve at $p$.

Lemma 1.1. Let $\mathcal{F}$ be an irreducible foliation in $\mathbb{P}^{2}$ of degree $m$, then the number of singular points, counted with multiplicity, is $m^{2}+m+1$.

For a proof see $[\mathbf{9}]$.

An algebraic solution of $\mathcal{F}$ is an irreducible algebraic curve $C$ that is the closure of a leaf of $\mathcal{F}$. Let $C$ be defined by $F\left(x_{0}: x_{1}: x_{2}\right)=0$. Then this can be expressed equivalently as:

a) An algebraic irreducible curve $C$ such that $F \mid d F \wedge \omega$.

b) An algebraic irreducible curve $C$ such that

$$
F \mid \sum_{i=0}^{2} Y_{i} \frac{\partial F}{\partial x_{i}}
$$

c) An algebraic irreducible curve $C$ such that $\alpha\left(\left.L_{-(m-1)}\right|_{C}\right) \subset T C$.

We say that $\mathcal{F}$ has a rational first integral if there exist a homogeneous rational fraction $R\left(x_{0}: x_{1}: x_{2}\right)$ such that $d R \wedge \omega=0$.

It can be proved that the following are equivalent:

i) $\mathcal{F}$ has an infinite number of algebraic solutions.

ii) All the solutions of $\mathcal{F}$ are algebraic. 
iii) All the solutions of $\mathcal{F}$ are the irreducible components of an irreducible pencil of plane curves $\lambda F+\mu G=0$ (i.e., for general $(\lambda, \mu)$ $\lambda F+\mu G=0$ is irreducible).

iv) $\mathcal{F}$ has a rational first integral. (For a proof see $[\mathbf{9}]$.)

The generic element of $\lambda F+\mu G=0$ is an irreducible curve of degree $d$. However, it is possible that for some finite number of $\left(\lambda_{i}: \mu_{i}\right)$ the corresponding curve is reducible or non-reduced

$$
\lambda_{i} F+\mu_{i} G=\prod_{j} U_{i j}^{n_{i j}}
$$

The values of $\left(\lambda_{i}: \mu_{i}\right)$ which have some $n_{i j}>1$ are called remarkable values.

By Bertini's Theorem the general member of this pencil is irreducible and non-singular away from the base locus, we call such a curve a generic solution of $\mathcal{F}$. We remark that the curves of the pencil $\lambda F+\mu G=0$ can be thought as the fibers of a rational map $\mathbb{P}^{2} \stackrel{f}{\rightarrow} \mathbb{P}^{1}$. The fact that the pencil is irreducible is equivalent to say that there does not exist a factorization of $f$ :

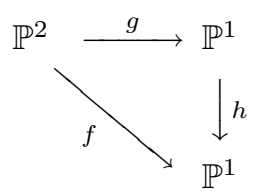

with $h$ of degree $>1$.

\section{Remarks.}

1. If $\mathcal{F}$ has a rational first integral, then integrating the associated local field in each singular point we see that its linear part must be diagonal and the quotients of its eigenvalues $u, v$ must be rational $(u / v \in \mathbb{Q})$. In the following by $(u, v)$ we denote relatively primes integer numbers. Moreover we suppose that $u \leq v$ and $u v \neq 0$, that is, the singularity is of multiplicity one. Following Poincaré $[\mathbf{1 0}]$ we adopt the following notation:

i) If $u / v<0$ the singularity is called a saddle.

ii) If $0<u / v<1$ the singularity is called a monocritical node.

iii) If $u / v=1$ the singularity is called a dicritical node.

2. Note that if $\mathcal{F}$ has a first integral then the singularities of the algebraic curves in the associated pencil are determinated by the 
pair $(u, v)$. More explicitly, if $C$ is a general solution of $\mathcal{F}$ and $\mathcal{F}$ has a node of type $(u, v)$ at $p$, then the singularity of $C$ at $p$ is analytically equivalent to $\prod_{j=1}^{r}\left(y^{u}-a_{j} x^{v}\right), a_{j} \in \mathbb{C}$ (see $\left.[\mathbf{1 0}]\right)$. A basic fact is:

$$
d F . G-F . d G=\prod_{i, j} U_{i j}^{n_{i j}-1} \omega
$$

and from this it follows at once that:

$$
2 d-2=\sum d_{i j}\left(n_{i j}-1\right)+m,
$$

where $\operatorname{deg} U_{i j}=d_{i j}$.

We give here another, more geometric proof of (1.1).

Lemma 1.2. Let $\mathcal{F}$ be a foliation in $\mathbb{P}^{2}$ of degree $m$. Suppose that $\mathcal{F}$ admits a first integral, and the generic solutions are curves of degree $d$, then

$$
2 d-2=\sum d_{i j}\left(n_{i j}-1\right)+m .
$$

Proof: Consider a line $L$ in $\mathbb{P}^{2}$ such that it does not pass through any singular point of $\mathcal{F}$ and is not tangent to any non-generic solution. To each point of $L$ correspond a unique value of $(\lambda: \mu)$, thus we have a rational map

$$
i: \mathbb{P}^{1} \rightarrow \mathbb{P}^{1},
$$

then the Riemann Hurwitz formula says $-2=-2 d+\sum d_{i j}\left(n_{i j}-1\right)+m$, where the ramifications are given by the remarkable values and the $m$ points of tangency of $L$ with the solutions of $\mathcal{F}$.

\section{Notation.}

1. In the following by a foliation $\mathcal{F}$ in $\mathbb{P}^{2}$ we understand an irreducible foliation with all its singular points of multiplicity one and with eingenvalues $\left(u_{i}, v_{i}\right)$, where $u_{i}, v_{i}$ denote relatively primes integers, $u_{i} \leq v_{i}$.

2. A foliation as above with all its nodes dicritical is called a dicritical foliation.

We recall that more generally we have:

Definition 4. Let $S$ be a complex algebraic surface (irreducible, reduced and non-singular). A foliation $\mathcal{F}$ in $S$ is given by a non-identically zero morphism of vector bundles

$$
\alpha: L \rightarrow T S
$$


where $L$ is a line bundle in $S$ and $T S$ is the tangent bundle of $S . \mathcal{F}$ has a rational first integral if the closure of the leaves are algebraic curves or, equivalently, if there exist a rational map $S \stackrel{\beta}{\rightarrow} X$, with $X$ a Riemann surface, such that the fibers of $\beta$ are the leaves of $\mathcal{F}$.

Since all the arguments and definitions about singular points of foliations in $\mathbb{P}^{2}$ are local, they can be extended at once to the case of algebraic surfaces.

\section{Reformulation of the Poincaré problem}

One of the fundamental results relating the degree of $\mathcal{F}$ and the nature of its algebraic solutions is the following proposition (this result was proved by Poincaré $[\mathbf{1 0}]$ in the case $S=\mathbb{P}^{2}$, our argument is a generalization of one due to Cerveau and Lins-Neto $[\mathbf{3}]$ ).

Proposition 2.1. Let $\mathcal{F}$ be a foliation on $S$ with a first integral and singular points of multiplicity one. Denote the pair of relatively prime integers associated to the eigenvalues of the nodes of $\mathcal{F}$ by $\left(u_{i}, v_{i}\right)$, then we have:

$$
\begin{gathered}
\sum_{i=1}^{n} r_{i}\left(u_{i}+v_{i}\right)=-C \cdot\left(K_{S}+L\right), \\
g=-\frac{C . L}{2}-\frac{\sum r_{i}}{2}+1 .
\end{gathered}
$$

Where $C$ is a general solution of $\mathcal{F}$,

$g=$ geometric genus of $C$,

$\alpha: L \rightarrow T S$ defines $\mathcal{F}$,

$K_{S}=$ canonical divisor of $S$,

$r_{i}=$ number of branches of $C$ through a node of $\mathcal{F}$,

$n=$ number of nodes of $\mathcal{F}$.

Proof: Let $\tilde{C} \stackrel{\pi}{\rightarrow} C$ be the normalization of $C$, the pullback of the restriction $\alpha\left(\left.L\right|_{C}\right)$ define a morphism $\tilde{\alpha}:\left.L\right|_{\tilde{C}} \rightarrow T \tilde{C}$ which is not injective in the $r_{i}$ points on the nodes $P_{i}$. This gives a section $s \in H^{0}\left(\tilde{C},\left.T \tilde{C} \otimes L^{-1}\right|_{\tilde{C}}\right)$ with $\sum_{i=1}^{n} r_{i}$ zeros, thus

$$
\sum_{i=1}^{n} r_{i}=2-2 g-C . L .
$$


Now, an easy generalization of the argument used in [8, p. 279-280], to compute the genus of a plane curve having simple singularities prove that if an embedded curve $C$ has singularities equivalent to $\prod_{j=1}^{r_{i}}\left(y^{u_{i}}-a_{j} x^{v_{i}}\right)$ then the geometric genus $g(C)$ is given by:

$$
2 g-2=C . C+C . K_{S}-\sum r_{i}^{2} u_{i} v_{i}+\sum r_{i}\left(u_{i}+v_{i}-1\right) .
$$

Combining (2.1) and (2.2) and C.C $=\sum_{i=1}^{n} r_{i}^{2} u_{i} v_{i}$ we get (1).

To prove (2), it is sufficient to evaluate in the genus formula the relation obtained in (1).

Remark. If $S=\mathbb{P}^{2}$, the formulas in 2.1 are:

(1) $\sum r_{i}\left(u_{i}+v_{i}\right)=(m+2) d$.

(2) $2 g-2=(m-1) d-\sum r_{i}$.

The Poincaré problem does not have a solution as formulated in the introduction. Here we present two examples:

Example 2.1. Consider the foliation in $\mathbb{P}^{2}$ given by the solutions of

$$
p y z . d x+q x z . d y-(p+q) y x . d z=0
$$

with $p, q$, positive integers, it is easy to see that any curve of the pencil

$$
\lambda x^{p} y^{q}+\mu z^{(p+q)}=0
$$

is a solution of (2.3). We can choose $d=p+q$ arbitrarily large and the foliation (2.3) is of degree 1.

Example 2.2. Consider the pencil of algebraic curves defined in affine coordinates by

$$
y^{d}=\lambda x^{d-2}(x-1)(y-1)
$$

We have:

\section{Proposition 2.2.}

(1) The generic element of (2.4) is irreducible.

(2) The foliation associated to (2.4) has degree 2 and all its singular points are of multiplicity one.

(3)

$$
g= \begin{cases}\frac{d-1}{2}, & \text { if } d \text { is odd } \\ \frac{d-2}{2}, & \text { if } d \text { is even }\end{cases}
$$


Proof: In fact, if the general element of (2.4) is reducible, then $h_{\lambda}(x, y)=y^{d}-\lambda x^{d-2}(x-1)(y-1)=f(x, y) g(x, y)$, thus $f(x, y)=$ $y^{p}+f_{1}(x, y), g(x, y)=y^{q}+g_{1}(x, y)$, with $p+q=d$.

But $h_{\lambda}$ has only two addends in which $y$ appear, $y^{d}$ and $\lambda y x^{d-2}(x-1)$, thus we must have $p=d, q=0$ and then $g(x, y)$ will be a constant. This proves moreover that the unique reducible curves in (2.4) are $y^{d}$ and $x^{d-2}(x-1)(y-1)$.

(2) follows by Lemma 1.2:

$$
m=2 d-2-(d-1)-(d-3)=2 .
$$

That all the points are simple is a count on the number of singularities of $\mathcal{F}$ (see Lemma 1.1).

In order to apply Proposition 2.1 to calculate the genus note that the point $(1,0)$ and the intersection at infinity of $y=0$ and $y=1$ are nodes with eigenvalues $(1, d)$ and the general curve is non-singular in these points, thus $r=1$. On the other hand $(0,0)$ is a node with eigenvalues $(d-2, d)$, if $d$ is odd and $((d-2) / 2, d / 2)$ if $d$ is even. In the last case $r=2$.

Then, it is necessary to reformulate the Poincaré problem. There are some options:

Pa) To bound $d$ in terms of $m$ and the eigenvalues of the nodes of $\mathcal{F}$ (note that in the precedings examples $d \leq \sum v_{i}$ ).

$\mathrm{Pb}$ ) From the formula $\sum r_{i}\left(u_{i}+v_{i}\right)=(m+2) d$, we see that in order to solve problem $\mathrm{Pa}$ ) it suffices to bound $\sum r_{i}$ in terms of $m$.

To solve this version of the problem we must imposes some conditions on the solutions of $\mathcal{F}$.

In fact, the following example shows that $\mathrm{Pb}$ ) has no solution in general:

Example 2.3. Consider the pencil of algebraic curves given by

$$
y^{d}(y-1)+\lambda x^{d}(x-1)=0 .
$$

The associated foliation has degree $m=2$ and a dicritical node in $(0,0)$. In this point the general curve has $d$ branches, so that $r=d$.

Remark. Proposition 2.1 has a very nice consequence. First note that

$$
(m+2) d=\sum r_{i}\left(u_{i}+v_{i}\right) \geq \sum r_{i}(u+v),
$$


where $(u+v)=\min \left(u_{i}+v_{i}\right)$, thus

$$
\sum r_{i} \leq \frac{(m+2) d}{u+v}
$$

On the other hand

$$
\begin{aligned}
& g=\frac{m-1}{2} d-\frac{\sum r_{i}}{2}+1 \geq \frac{m-1}{2} d-\frac{m+2}{2(u+v)} d+1, \\
& g \geq \frac{m(u+v-1)-(u+v+2)}{2(u+v)} d+1 .
\end{aligned}
$$

Hence, if $m(u+v-1)>(u+v+2)$ a bound for the genus is a bound for the degree $d$. The above condition is fulfilled unless

(1) $m=1$,

(2) $m=2$ and $(u+v) \leq 4$,

(3) $m=3$ and $u+v=2$,

(4) $m=4$ and $(u+v)=2$.

Thus, to solve the refomulated Poincaré problem in most of the cases, it suffice to bound the genus of the generic solution by means of some information depending of $\mathcal{F}$. This allows us to work with any curve birationally equivalent to $C$.

The following proposition give a partial solution to the reformulated Poincaré problem. Let $C$ be a generic solution of $\mathcal{F}$ and let $\pi: S \rightarrow \mathbb{P}^{2}$ be a chain of blowing-ups such that the total transform of $C$ is a curve with normal crossing. So, if we call $\tilde{C}$ the proper transform of $C$ under $\pi$, $\tilde{C}$ is the embedded desingularization of $C$. We say that the plane curves $C$ and $C^{\prime}$ of the same degree $d$, are linearly equisingulars if under the minimal chain of blowing-ups that desingularize $C, C^{\prime}$ has the following behavior:

1. The proper transform of $C^{\prime}$ under each blowing-up of the chain has singularities in the same points that the proper transform of $C$ and these singularities have the same multiplicities.

2. The proper transform of $C^{\prime}$ under $\pi$ is the minimal embedded resolution of $C^{\prime}$.

This is a somewhat stronger form of the usual concept of topological equisingularity. It follows easily from the structure of the Picard variety of the blowing-up, that if $\tilde{C}$ and $\tilde{C}^{\prime}$ denotes respectively the proper 
transforms of $C$ and $C^{\prime}$ under $\pi$, then $\mathcal{O}_{S}(\tilde{C}) \stackrel{\sim}{\longrightarrow} \mathcal{O}_{S}\left(\tilde{C}^{\prime}\right)$, this equivalence justify the terminology. In others words, the closure of the set of plane curves linearly equisingulars to $C$ is a linear system.

If $C^{\prime}$ is another general solution of $\mathcal{F}$, then $C^{\prime}$ is linearly equisingular to $C$ and we shall denote

$$
\delta=\operatorname{deg}\left(\mathcal{O}_{\tilde{C}}(\tilde{C})\right)=\sum_{u_{i}=r_{i}=1} v_{i}
$$

that is, the sum is taken on the nodes of $\mathcal{F}$ that are non-singular points of $C$.

Proposition 2.3. If the conditions of being linearly equisingular to $C$ imposes independent conditions on curves of degree $d$ then

$$
g(C) \leq \delta .
$$

Proof: First note that the linear system of curves linearly equisingulars to $C$ restricted to $\tilde{C}$ is equivalent to the linear system $\mathcal{O}_{\tilde{C}}(\tilde{C})$. Now, the dimension of the linear system $|\tilde{C}|$ can be calculated in two differents ways (see $[8$, p. 713$]$ ):

1) It is the dimension of the linear system of plane curves of degree $d$ being linearly equisingulars to $C$. Thus, its dimension is:

$$
\operatorname{dim}|\tilde{C}|=\frac{d(d+3)}{2}-\frac{\sum r_{p}\left(r_{p}+1\right)}{2}+w,
$$

where the sum is over all the singular points of $C$ (including the infinitely near singular points), and $w$ is the number of these linear conditions being non independent.

2) It is the dimension of the space of global sections of $\mathcal{O}_{S}(\tilde{C})$. From the exact sequence

$$
0 \rightarrow \mathcal{O}_{S} \rightarrow \mathcal{O}_{S}(\tilde{C}) \rightarrow \mathcal{O}_{\tilde{C}}(\tilde{C}) \rightarrow 0
$$

and the fact that $S$ is rational we have

$$
h^{0}\left(\tilde{C}, \mathcal{O}_{\tilde{C}}(\tilde{C})\right)=h^{0}\left(S, \mathcal{O}_{S}(\tilde{C})\right)-1,
$$

and

$$
h^{1}\left(\tilde{C}, \mathcal{O}_{\tilde{C}}(\tilde{C})\right)=h^{1}\left(S, \mathcal{O}_{S}(\tilde{C})\right) .
$$

Thus, using the Riemann-Roch Theorem we obtain:

$$
\begin{aligned}
\operatorname{dim}|\tilde{C}| & =h^{0}\left(S, \mathcal{O}_{S}(\tilde{C})\right)-1=h^{0}\left(\tilde{C}, \mathcal{O}_{\tilde{C}}(\tilde{C})\right) \\
& =\delta-g+1+h^{1}\left(\tilde{C}, \mathcal{O}_{\tilde{C}}(\tilde{C})\right) .
\end{aligned}
$$

Comparing the two formulas above and using $\delta=d^{2}-\sum r_{p}^{2}$, we obtain $w=h^{1}\left(\tilde{C}, \mathcal{O}_{\tilde{C}}(\tilde{C})\right)$. So, under our hypothesis we have $h^{1}\left(\tilde{C}, \mathcal{O}_{\tilde{C}}(\tilde{C})\right)=0$. We obtain then, $g \leq \delta$, since $\operatorname{dim}|\tilde{C}| \geq 1$. 


\section{Dicritical foliations}

It is well know that the general pencil of plane curves of degree $d$ is a pencil in which two general members are non-singular curves with transversal intersection. There is a natural question, how to identify when an algebraic foliation in $\mathbb{P}^{2}$ has such a generic pencil as a first integral?

Some immediate necessary conditions are: all the nodes of $\mathcal{F}$ must be dicritical and all the saddles with eigenvalues $(1,-1)$. In this section we investigate if these conditions are sufficient, that is, if $\mathcal{F}$ has a first integral, all its nodes are dicritical and all its saddles have eigenvalues $(1,-1)$, then is the first integral determined by a generic pencil? This problem can be stated on a arbitrary algebraic surface $S$, we study it when $S$ is a rational surface.

In this section by "the fiber of a rational map" we understand the scheme theoretical fiber of the morphism $f$. Thus, for example, if we express a pencil of plane curves $\lambda F+\mu G=0$ as a rational map $\mathbb{P}^{2} \stackrel{r}{\rightarrow} \mathbb{P}^{1}$ the fiber of $\left(\lambda_{0}, \mu_{0}\right)$ will be the, possibly non-reduced, curve $\lambda_{0} F+\mu_{0} G=$ 0 with this convention we have:

Proposition 3.1. Let $f: S \rightarrow \mathbb{P}^{1}$ be a rational map between the rational surface $S$ and $\mathbb{P}^{1}$. If there exist $t_{1}, t_{2}, t_{3}$ such that $f^{-1}\left(t_{i}\right)=n_{i} E_{i}$ is a multiple divisor $\left(n_{i}>1\right)$, then all the fibers of $f$ are reducible curves.

Proof: Let $f^{-1}\left(t_{i}\right)=n_{i} E_{i},\left(E_{i}\right.$ possibly reducible) $\bar{S}=S-\cup\left(E_{i}\right)$, $\overline{\mathrm{P}}^{1}=\mathbb{P}^{1}-\left\{t_{i}\right\}$.

Let $\mathcal{P}$ be either of the following: $\mathbb{P}^{1}, \mathbb{C}$, or $\mathbb{H}$, the upper half plane. Depending on $\frac{1}{n_{1}}+\frac{1}{n_{2}}+\frac{1}{n_{3}}$ being $>$, = or $<$ than 1 we can construct a triangle $\Delta$ on the suitable $\mathcal{P}$, such that the amplitude of the interior angles of $\Delta$ are $\frac{\pi}{n_{i}}$. Let $G$ be the triangular group on $\mathcal{P}$ associated to $\Delta$, by a theorem of Fox ([4]) there exist a normal subgroup $\bar{G} \subset G$ of finite index, and thus an algebraic curve $X(=\mathcal{P} / \bar{G})$ and a regular map

$$
X \stackrel{g}{\rightarrow} \mathbb{P}^{1}
$$

ramified on three values (we can suppose that these are the $t_{i}$ ), such that near each branch point $g$ is given by $z^{n_{i}}$. Let $\tilde{X}=X-g^{-1}\left(t_{i}\right)$. We have

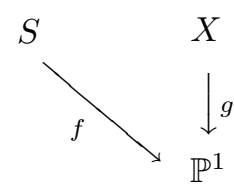


Our goal is to construct a rational map $S \stackrel{h}{\rightarrow} X$ such that $g \circ h=f$.

To start with fix a point $p_{0} \in \bar{S}$, let $f\left(p_{0}\right)=t_{0}$ and choose $s_{0}$ such that $s_{0} \in g^{-1}\left(t_{0}\right)$. The construction of $h$ will be the following: take $p \in S$ and consider a path joining $p_{0}$ and $p$, project it by means of $f$ and lift it to $\tilde{X}$ by means of $g$. The ending point of this lifting, starting from $s_{0}$ will be $s=h(p)$.

In order to prove that $h$ is well defined we must show that this construction sends closed paths into closed paths.

Let $\gamma \in \pi_{1}\left(\bar{S}, p_{0}\right)$. It follows by a theorem of Deligne ([5, Theorem 1.1(B)]) that given a birational map $S \stackrel{\alpha}{\longrightarrow} \mathbb{P}^{2}$ there exist a generic line $l$ in $\mathbb{P}^{2}$ such that if $L=\alpha^{-1}(l)$ then there exist $\gamma_{L} \in \pi_{1}\left(L-L \cap\left\{E_{i}\right\}\right)$ such that $\gamma \sim \gamma_{L}$. If $\gamma_{L}$ is the union of closed paths that go once around points of $L \cap\left\{E_{i}\right\}$ then the image of $\gamma_{L}$ is a closed path that go $n_{i}$ times around some $t_{i}$, because near a point of $L \cap\left\{E_{i}\right\} f$ is of the form $z^{n_{i}}$. As $g$ is locally $z^{n_{i}}$ the image of $\gamma_{L}$ lift to a closed path that go once around the corresponding point in $g^{-1}\left(t_{i}\right)$. A similar argument work out if $\gamma_{L}$ is expressed as a linear combination of the standard basis of $\pi_{1}\left(L-L \cap\left\{E_{i}\right\}\right)$. This prove the existence of $h$. That $h$ is rational follows at once because $f=g \circ h$ and $h$ has finite fibers.

Proposition 3.2. Let $\mathcal{F}$ be a dicritical foliation in a rational surface $S$ with an irreducible rational first integral and $C$ a reducible fiber of the associated irreducible pencil. Consider $\bar{S}=S$-\{nodes of $\mathcal{F}\}$ and $\bar{C}$ the restriction of $C$ to $\bar{S}$. Then $\bar{C}$ is connected.

Proof: Denote by $P_{1}, \ldots, P_{n}$ the nodes of $\mathcal{F}$ and consider the rational surface $\tilde{S}$ obtained by blowing up $S$ in the $n$ nodes.

The proper transform $\tilde{\mathcal{F}}$ of $\mathcal{F}$ is then a foliation that have as invariant curves just the proper transform of the solutions of $\mathcal{F}$. Suppose that for $C$ as in the hypothesis $\bar{C}$ is disconnected. As the possible selfintersections of $C$ in the nodes disappears after the blow ups, the proper transform $\tilde{C}$ of $C$ must be disconnected.

On the other hand the induced pencil

$$
\tilde{S} \stackrel{\tilde{f}}{\rightarrow} \mathbb{P}^{1}
$$

has a generic irreducible fiber (because $\underset{\tilde{f}}{\stackrel{f}{\rightarrow}} \mathbb{P}^{1}$ is irreducible). By the Zariski Lemma ([1] ) all the fibers of $\tilde{f}$ are connected which gives a contradiction.

A first consequence of the Proposition 3.2 is that if all the saddles of a dicritical foliation are of the form $(-1,1)$ then all the fibers of $f$ with 
some multiple component must be a power of a reduced curve (as -1 is the quotient of the multiplicities of the components of the corresponding fiber having intersection in this point). Combining 3.1 and 3.2 we have:

Proposition 3.3. Let $\mathcal{F}$ be a dicritical foliation in a rational surface $S$ with singularities of multiplicity one. If $\mathcal{F}$ has an irreducible rational first integral $S \stackrel{f}{\rightarrow} \mathbb{P}^{1}$ and all its saddle points have eigenvalues $(1,-1)$ then there are at most two fibers of $f$ with some multiple component and they are powers of reduced curves.

Now we can prove:

Theorem 3.4. Let $\mathcal{F}$ be a irreducible foliation in a rational surface $S$ with singularities of eigenvalues $(1,1)$ or $(1,-1)$. If $\mathcal{F}$ has a rational first integral $f: S \rightarrow \mathbb{P}^{1}$, then all the fibers of $f$ are reduced curves and the general one is a non-singular curve meeting transversally any other fiber of $f$.

Proof: The main idea is to look at the local structure of the pencil near a dicritical node $P$. If the general curve of the pencil has $r$ different tangents at $P$, then we have defined a regular map $\mathbb{P}^{1} \stackrel{h}{\rightarrow} \mathbb{P}^{1}$ of degree $r$, which send each direction through $P$ to the value of the corresponding curve in $\mathbb{P}^{1}$ via $f$.

By Riemann-Hurwitz we have:

$$
2 r-2=\sum_{i=1}^{k} r_{i}\left(n_{i}-1\right)
$$

where the sum is on the multiple components of the pencil, $n_{i}$ the corresponding multiplicity and $r_{i}$ the number of branches of each multiple curve at $P$.

Using Proposition 3.3

$$
\begin{gathered}
2 r-2=2 r-\sum_{i=1}^{k} r_{i}, \\
\sum_{i=1}^{k} r_{i}=2 .
\end{gathered}
$$

Then we must have $k=2, r_{1}=r_{2}=1$. Thus there are only two ramification points, which implies $n_{1}=n_{2}$, but if $n_{1}=n_{2}>1$ then there exist a commutative diagram 


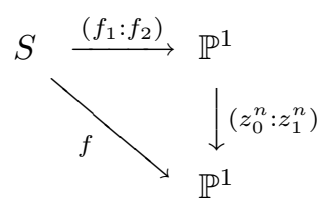

$\left(f_{1}^{n}, f_{2}^{n}\right.$ the corresponding multiple fibers of $f$ ), and thus the pencil given by $f$ is not irreducible.

We conclude that $n_{1}=n_{2}=1$ that is, $h$ is unramified and then $r=1$ and the general curve has only one branch through $P$.

Note that using Lemma 1.2 we have obtained:

Corollary 3.5. Let $\mathcal{F}$ be a foliation in $\mathbb{P}^{2}$ of degree $m$ with a first integral of degree $d$, if all the singular points of $\mathcal{F}$ have eigenvalues $(1,1)$ or $(1,-1)$ then

$$
m=2 d-2 .
$$

\section{References}

1. W. Barth, C. Peters and A. Van de Ven, "Compact complex surfaces," Springer Verlag, 1984.

2. M. CARnicer, The Poincaré problem in the non-dicritical case, Ann. of Math. 140(2) (1994), 289-294.

3. D. Cerveau and A. Lins Neto, Holomorphic foliations in $\mathbb{C} P(2)$ having an invariant algebraic curve, Ann. Inst. Fourier (Grenoble) 41 (1991), 883-903.

4. R. H. Fox, On Frenchel's conjecture about F-groups, Mat. Tidsskrift B (1952), 61-65.

5. W. Fulton, On the topology of algebraic varieties, Proc. Amer. Math. Soc. 46 (1987), 15-46.

6. X. Gómez-Mont And L. Ortiz-Bobadilla, "Sistemas dinámicos holomorfos en superficies," Aportaciones Matemáticas, Sociedad Matemática Mexicana, 1988.

7. X. Gómez-Mont and R. Vila, On meromorphic integrals of holomorphic foliations in surfaces, unpublished.

8. P. GRIfFiths And J. HARRIS, "Principles of algebraic geometry," Wiley Interscience, 1978.

9. J. P. Jounalou, "Equations de Pfaff algébriques," Lecture Notes in Mathematics 708, Springer Verlag, 1979. 
10. H. Poincaré, Sur l'intégration algébrique des équations différentielles du premier order et du premier degré, Rend. Circ Mat. Palermo 5 (1891), 161-191.

11. H. Poincaré, Sur l'intégration algébrique des équations différentielles du premier ordre et du premier degré, Rend. Circ. Mat. Palermo 11 (1897), 193-239.

\author{
Instituto de Matemáticas \\ UNAM \\ Unidad Morelia \\ Nicolás Romero 150 \\ Colonia Centro Morelia \\ Michoacán \\ MEXICO \\ e-mail: amgarcia@jupiter.ccu.umich.mx
}

Primera versió rebuda el 6 de Novembre de 1995, darrera versió rebuda el 23 de Gener de 1997 